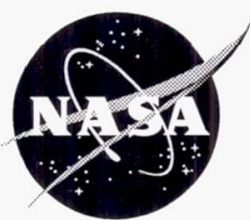

Modeling of Transmittance Degradation Caused by Optical Surface Contamination by Atomic Oxygen Reaction With Adsorbed Silicones

Aaron Snyder, Bruce Banks, and Sharon Miller

Glenn Research Center, Cleveland, Ohio

Thomas Stueber and Edward Sechkar

Dynacs Engineering Company, Inc., Brook Park, Ohio 
Since its founding, NASA has been dedicated to the advancement of aeronautics and space science. The NASA Scientific and Technical Information (STI) Program Office plays a key part in helping NASA maintain this important role.

The NASA STI Program Office is operated by Langley Research Center, the Lead Center for NASA's scientific and technical information. The NASA STI Program Office provides access to the NASA STI Database, the largest collection of aeronautical and space science STI in the world. The Program Office is also NASA's institutional mechanism for disseminating the results of its research and development activities. These results are published by NASA in the NASA STI Report Series, which includes the following report types:

- $\quad$ TECHNICAL PUBLICATION. Reports of completed research or a major significant phase of research that present the results of NASA programs and include extensive data or theoretical analysis. Includes compilations of significant scientific and technical data and information deemed to be of continuing reference value. NASA's counterpart of peerreviewed formal professional papers but has less stringent limitations on manuscript length and extent of graphic presentations.

- TECHNICAL MEMORANDUM. Scientific and technical findings that are preliminary or of specialized interest, e.g., quick release reports, working papers, and bibliographies that contain minimal annotation. Does not contain extensive analysis.

- CONTRACTOR REPORT. Scientific and technical findings by NASA-sponsored contractors and grantees.
- CONFERENCE PUBLICATION. Collected papers from scientific and technical conferences, symposia, seminars, or other meetings sponsored or cosponsored by NASA.

- SPECIAL PUBLICATION. Scientific, technical, or historical information from NASA programs, projects, and missions, often concerned with subjects having substantial public interest.

- TECHNICAL TRANSLATION. Englishlanguage translations of foreign scientific and technical material pertinent to NASA's mission.

Specialized services that complement the STI Program Office's diverse offerings include creating custom thesauri, building customized data bases, organizing and publishing research results ... even providing videos.

For more information about the NASA STI Program Office, see the following:

- Access the NASA STI Program Home Page at http://www.sti.nasa.gov

- E-mail your question via the Internet to help@sti.nasa.gov

- Fax your question to the NASA Access Help Desk at 301-621-0134

- Telephone the NASA Access Help Desk at 301-621-0390

- Write to: NASA Access Help Desk NASA Center for AeroSpace Information 7121 Standard Drive Hanover, MD 21076 
NASA/TM-2001-210597

\section{Modeling of Transmittance Degradation Caused by Optical Surface Contamination by Atomic Oxygen Reaction With Adsorbed Silicones}

Aaron Snyder, Bruce Banks, and Sharon Miller

Glenn Research Center, Cleveland, Ohio

Thomas Stueber and Edward Sechkar

Dynacs Engineering Company, Inc., Brook Park, Ohio

Prepared for the

45th Annual Meeting, the International Symposium on Optical Science and Technology sponsored by the International Society for Optical Engineering

San Diego, California, July 30-August 4, 2000

National Aeronautics and

Space Administration

Glenn Research Center 
Available from

NASA Center for Aerospace Information 7121 Standard Drive

Hanover, MD 21076
National Technical Information Service 5285 Port Royal Road Springfield, VA 22100

Available electronically at http://gltrs.grc.nasa.gov/GLTRS 


\title{
Modeling of Transmittance Degradation Caused by Optical Surface Contamination by Atomic Oxygen Reaction With Adsorbed Silicones
}

\author{
Aaron Snyder," Bruce Banks, and Sharon Miller \\ National Aeronautics and Space Administration \\ Glenn Research Center \\ Cleveland, Ohio 44135 \\ Thomas Stueber and Edward Sechkar \\ Dynacs Engineering Company, Inc. \\ Brook Park, Ohio 44142
}

\begin{abstract}
A numerical procedure is presented to calculate transmittance degradation caused by contaminant films on spacecraft surfaces produced through the interaction of orbital atomic oxygen ( $\mathrm{AO}$ ) with volatile silicones and hydrocarbons from spacecraft components. In the model, contaminant accretion is dependent on the adsorption of species, depletion reactions due to gas-surface collisions, desorption, and surface reactions between $\mathrm{AO}$ and silicone producing $\mathrm{SiO}_{\mathrm{x}}$ (where $\mathrm{x}$ is near 2). A detailed description of the procedure used to calculate the constituents of the contaminant layer is presented, including the equations that govern the evolution of fractional coverage by specie type. As an illustrative example of film growth, calculation results using a prototype code that calculates the evolution of surface coverage by specie type is presented and discussed. An example of the transmittance degradation caused by surface interaction of AO with deposited contaminant is presented for the case of exponentially decaying contaminant flux. These examples are performed using hypothetical values for the process parameters.
\end{abstract}

\section{INTRODUCTION}

Surface contamination of components continues to be a primary concern with regard to maintaining performance levels of spacecraft in low Earth Orbit (LEO). ${ }^{1-4}$ As recent as the $8^{\text {th }}$ International Symposium On "Materials in a Space Environment" held in Arcachon, France during June 2000, it was determined to be the problem of most widespread interest according to a survey response. Particular contaminants of interest include silicones and hydrocarbons released from spacecraft components. ${ }^{5}$ Silicones are widely used as potting compounds, adhesives, seals, gaskets, hydrophobic surfaces, and atomic oxygen $(\mathrm{AO})$ protective coatings. Some of these silicones are volatile. Volatile silicone molecules can arrive at and deposit on exposed spacecraft surfaces along with volatile hydrocarbons. Some of these surfaces are also exposed to AO that interacts with and oxidizes deposited silicones and hydrocarbons. It is believed that some of the oxidation products contribute to the formation of contaminant films, while others are volatile products. Contaminant films pose a risk to spacecraft performance because they can act as an optically absorbing film on the surfaces of sun sensors, star trackers, and optical components or increase the solar absorptance of thermal control surfaces. This paper addresses the attenuation of optical sensor transmittance caused by an accumulation of surface contaminants formed by the reaction of in-space AO with volatile silicones and hydrocarbons discharged from spacecraft materials.

$\mathrm{AO}$ is abundant in the LEO environment and readily reacts chemically with many exposed organic materials during a mission. A chief product of such a reaction with silicone-containing materials is $\mathrm{SiO}_{\mathrm{x}}$. To help predict the effects of the space environment interaction with organic compounds to form contaminant film on optical surfaces, a numerical model was constructed. In the model, specified silicone and hydrocarbon molecular fluxes strike a fused silica substrate and are partially adsorbed. Some of the adsorbed species chemically react with a concurrent specified AO flux. The model is based on a double-layer (surface layer and first sub-layer) bookkeeping procedure that relates the respective fractional surface areas of the participating species to parameters including volatile species flux, surface adsorption and desorption coefficients, and AO chemical reaction probability. Because of sublation through desorption and other depletion mechanisms that partially expose the sub-layer, the fractional species coverage is also book-kept for the first sub-layer of adsorbates. Updating the relative surface coverage is dependent on the fractional surface and sub-layer compositions, on current species fluxes, and on the

\footnotetext{
*Correspondence: E-mail: aaron.snyder@grc.nasa.gov; Phone: 216-433-5918; Fax: 216-433-2221
} 
probabilities of adhesion, desorption, depletion and chemical reaction. Because surface adsorption even with only one species type is a complex process, and because the detailed interaction mechanisms are not clearly understood or readily calculated, many processes are treated in a simplified manner and include the use of hypothetical values for the process parameters.

Surface reactions are attributed to $\mathrm{AO}$ and silicone molecules present on the surface. Reactions can also occur due to the $\mathrm{AO}$ flux and silicone flux impinging on the surface sites occupied by these species types. It is assumed that the molecular $\mathrm{SiO}_{\mathrm{x}}$ product forms a surface coating which, depending on species coverage, traps other molecules beneath it. The trapped molecules that may influence optical absorptance are volatile silicone or hydrocarbon molecules. The contaminant film growth depends on the surface reaction of adsorbed AO and silicone molecules, on the reaction of AO flux with adsorbed silicone molecules, and on the reaction of silicone flux with adsorbed AO atoms. The accumulated contaminant layer is computed by modeling the accretion. The variation in optical transmittance (neglecting UV darkening effects) can be calculated at successive stages by comparing the surface portions of contaminant film due to accrued $\mathrm{SiO}_{\mathrm{x}}$ versus the surface portion due to trapped hydrocarbons and silicones.

\section{ADSORPTION AND DESORPTION CONCEPTS}

Depending on the energy of adsorption, surface adsorbate bonding is classified under two broad types: physisorbed or chemisorbed. ${ }^{6-7}$ Physisorption is associated with weaker intermolecular forces such as van der Waals interactions, whereas chemisorption is due to stronger covalent, ionic and metallic bonding caused by electron exchange between adsorbate and adsorbent. The weak physisorption bonds allow the adsorption process to be easily reversed, and hence average residence times for physisorbed molecules is short. Because adsorption is an exothermic process, the residence time, $\tau_{r}$, of a physisorbed molecule depends on the amount of excess energy it retains after being adsorbed. The residence time for a physisorbed or chemisorbed molecule can be related to the typical vibration period $\tau_{\mathrm{o}}\left(\sim 10^{-13} \mathrm{~s}\right)$ and to adsorption enthalpy, $\Delta \mathrm{h}_{\mathrm{a}}$, by the following Arrhenius type expression

$$
\tau_{r}=\tau_{o} e^{-\Delta h_{a} / R T}
$$

where $R$ is the gas constant and $T$ is the substrate temperature. Upon examining this expression, it is clear that the lifetime of an adsorbed molecule depends strongly on the potential well into which it is adsorbed and on the temperature. For example, at a temperature of $273 \mathrm{~K}$ and using energies of adsorption equal to $-30 \mathrm{~kJ} / \mathrm{mole}$ and $-100 \mathrm{~kJ} / \mathrm{mole}$ for physisorption and chemisorption, respectively, the corresponding residence times of $5.5 \times 10^{-8} \mathrm{~s}$ and $1.4 \times 10^{6} \mathrm{~s}$ are obtained. These times are many orders of magnitude apart. Furthermore, increasing the temperature to $373 \mathrm{~K}$ correspondingly results in decreasing the residence times to $1.6 \times 10^{-9} \mathrm{~s}$ and $10 \mathrm{~s}$, respectively. Often for a process, it can be assumed that equilibrium is achieved between adsorption and desorption over the time period of the chemisorption process. For our purposes, the chemisorption of silicones can be considered as being carried out in successive steps, as physisorption followed by activation to a bound state through its interaction with either adsorbed $\mathrm{AO}$ or $\mathrm{AO}$ flux. Desorption is also an activated process; here the flux of any component may activate desorption. In this paper the microscopic details of the mechanisms are not evaluated but macroscopic procedures are used based on probabilistic arguments to represent the gas-surface processes.

Because the typical time scale of physisorption is much smaller than the time of contamination (months or years in LEO) the details of individual atoms or molecules striking a surface are replaced by a net flux of molecules that, based on accompanying sticking probabilities, produces a coverage representative of a detailed balance approach. Consequently, the small time increments associated with microscopic events are linked together into much larger steps, which in turn may be small compared to the total experimental period. The sticking probability, $P_{s}$, for an adsorbate can be defined descriptively as

$$
P_{s}=\frac{\text { rate of adsorption of molecules at the surface }}{\text { rate of collisions of molecules with the surface }} .
$$

It is understood that $P_{s}$ itself may depend on coverage since it is dependent on $\Delta h_{a}$, which depends on surface coverage. Thus, the rates of adsorption and desorption are highly influenced by the activation energy barrier, which depends on surface coverage. At a particular instant, the existing surface will be covered with various species that may be randomly distributed or perhaps distributed as clusters of various single specie islands. Although the particular arrangement of adsorbed molecules affects the thermodynamics of the process, effects of site arrangement are not addressed in this paper. 


\section{FRACTIONAL SURFACE COVERAGE AND SUB-LAYER COVERAGE}

For conceptual purposes it is convenient to group the various surface species into contiguous regions. This is also useful in bookkeeping the different processes. Accordingly, a schematic drawing of the surface coverage two layers deep is given in figure 1. This schematic indicates that the upper surface is composed of the four species, with the fractional coverage given by $F_{g}, F_{s}, F_{h c}$ and $F_{a o}$ for the silica-glass, silicone, hydrocarbon and $\mathrm{AO}$, respectively. These are the four larger rectangles that constitute the unit area. The coverage components of the first sub-layer are also indicated and denoted by $F^{i}$, where both indices run over the species range. Thus, for example, the second small rectangle from the left along the top row is denoted $F^{g}{ }_{s}$ and indicates the fraction of glass in the top layer that is covering silicone. From this definition, it follows that the sum of $F^{g}{ }_{g}, F_{s}^{g}, F_{h c}^{g}$ and $F_{a o}^{g}$ equals one because they constitute the four sub-layer fractions covered by glass. These fractional components are multiplied by $F_{g}$ to obtain equivalent sub-layer fractions of the total unit area (not just the glass area). This holds for the fractional coverage below the other species. The connection between the sub-layer fraction in terms of coverage specie and the sub-layer fraction in terms of total area is given by the following equation

$$
f_{j}^{i}=F_{i} F_{j}^{i} \quad \text { with } \quad \sum_{j} F_{j}^{i}=1 \text { and } \sum_{j} f_{j}^{i}=F_{i} \text {, }
$$

where $f_{i}^{i}$ has been introduced to denote the fraction of the total unit area that sub-layer type $i$ covered by type $j$ represents. In what follows, the $F_{i}$ and $f_{j}^{i}$ are referred to as absolute fractions because they are fractions of the total unit area. Similarly, the $F^{i}{ }_{j}$ are called relative fractions because they are fractions of the non-unit $F_{i}$.

\section{RELATIVE CHANGE OF COVERAGE BASED ON THE PROCESS}

\subsection{Adsorption}

The fractional area of coverage can be computationally modeled by approaching it from the perspective of exposing a surface to a fluence burst of $m$ molecules per unit area. The following processes are based on a unit time period. In a subsequent section, the fractional coverage will be expressed in iterated form. Based on a sticking probability, $P_{s}$, and $n$ available discretely defined sites per unit area, the fractional coverage, $\theta$, is derived from simple statistical considerations, when allowing for multiple hits on a site, as

$$
\theta=1-e^{-\left(m_{i} P_{s} / n\right)}
$$

This provides a convenient form from which to obtain the change in species coverage given $m, n$, and $P_{s}$. For given surface conditions (fixed $n$ and $P_{s}$ ), it can be seen from Equation [4] that $\theta$ approaches unity for large atomic or molecular fluences. Figure 2 shows curves of fractional coverage versus fluence-to-site-density ratio for various $P_{s}$. At higher values of $P_{s}$, the curves become more nonlinear, indicative of a larger percentage of molecules hitting occupied sites. Equation [4] will be used to determine fractional coverage based on adsorption. It is assumed that $P_{s}$ is known or prescribed for every possible species-flux-on-surface combination.

Based on the preceding discussion, the relative fractional coverage due to the adsorption process is defined for the various flux-surface combinations. The relative fractional coverage due to each of the other processes is discussed subsequently. In this model, the three species of fluxes are $A O$, silicone, and hydrocarbon, which can land on sites occupied by the other species as well as on the silica glass substrate. Accordingly, the following sticking probabilities are used: $P_{i-j}^{a}$ where the superscript " $a$ " indicates adsorption (to distinguish the adsorption process from the other processes), the left subscript index " $i$ " represents the flux species (denoted specifically by the indices s, hc, ao), and the right subscript index " $j$ " represents the surface species (denoted specifically by the indices g, s, hc, ao). Using these sticking probabilities, the fractional coverage of a species " $i$ " on species " $j$ " is given by

$$
G_{i-j}^{a}=1-e^{-\left(m_{i} P_{i-j}^{a} / n\right)}
$$




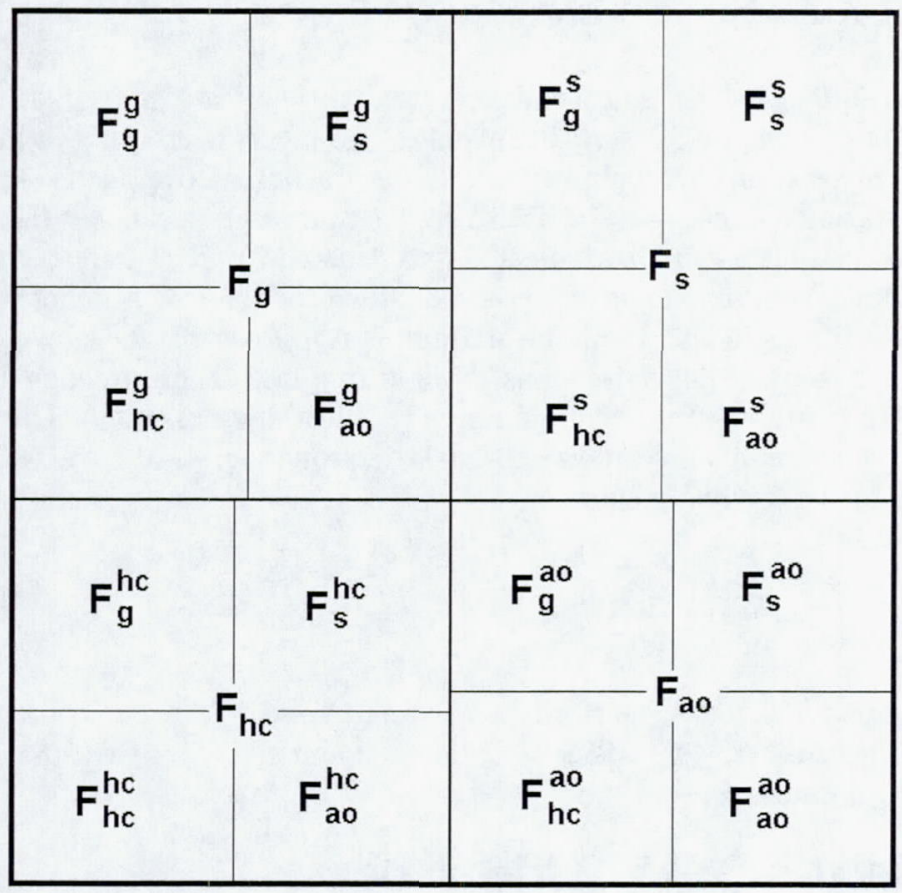

Fig. 1 - Two-level fractional coverage of unit area.

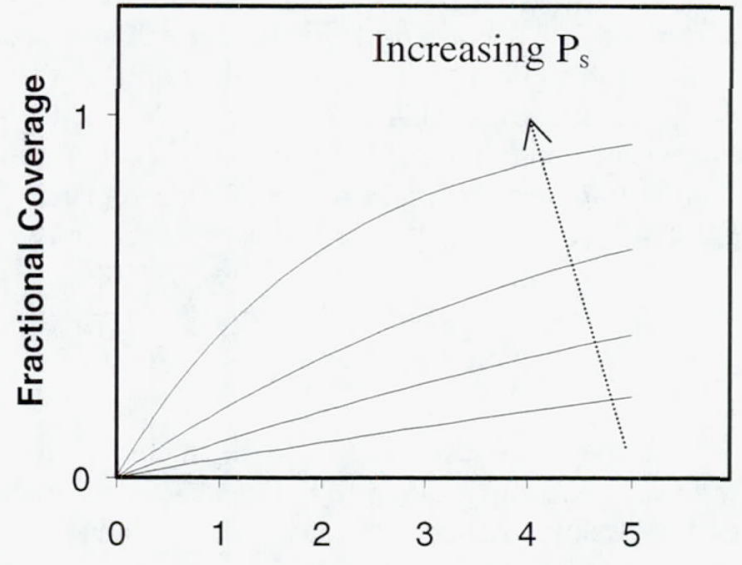

Fluence-to-Site-Density Ratio, $\mathrm{m} / \mathrm{n}$

Fig. 2 - Fractional coverage versus fluence for various $P_{s}$.

The quantity $G$ represents a percentage of surface species $j$ that is converted to species $i$ and, therefore, is a relative fraction of $F_{j}$. This representation holds for the other processes to be discussed. Using adsorption as the example, it is emphasized that the relative coverage produced is due to a fluence $m$ of species type $i$ striking type $j$ having $n$ sites per unit area; the site density $n$ includes all sites and remains fixed regardless of specie occupancy. Consequently, the resulting relative fractional coverage represents a change in absolute fractional coverage, brought about by the process occurring during the time interval associated with the prescribed fluence increment. The relative coverage brought about by the other processes is discussed in the following subsections.

\subsection{Desorption}

Since desorption is an activated process, it is dependent on substrate temperature. It is also dependent on surface coverage. As a substrate is heated, the rate of desorption may have a maximum due to greater difficulty in removing the more tightly bound molecules remaining at lower coverage. However, for a fixed coverage, the rate of desorption will generally be an increasing function of temperature. In the modeling, the processes are calculated assuming a fixed temperature. The fraction of type $i$ molecules being desorbed from type $j$ molecules in a unit time is denoted by $G^{d s}{ }_{i-j}$. One model used here for desorption is given by

$$
G_{i-j}^{d s}=e^{-c \Delta h_{d s} / R T}
$$

where $\mathrm{c}$ is a constant depending on the adsorbate and adsorbent combination. In this model, desorption occurs from the upper layer only.

\subsection{Reaction depletion by collision}

Atoms and molecules can also be removed from the surface due to reaction depletion. For example, AO striking hydrocarbon sites can cause the hydrocarbon to be activated sufficiently to escape the surface. This depletion mechanism is assumed to hold for all combinations except fluxes hitting glass sites, which are assumed inert to removal. The probability of type $j$ molecules being depleted by collisions with type $i$ flux in a unit time is denoted by $G^{r d}{ }_{i-j}$. Since the statistics for depletion 
follow identically from the statistics of adsorption, the expression for the depletion fraction is similar to equation [5] and is given by

$$
G_{i-j}^{r d}=1-e^{-\left(m_{i} P_{i-j}^{r d} / n\right)}
$$

where $P_{i j-j}^{r d}$ is the probability that a site is depleted given an $i$-type fluence $m_{i}$ and overall site density $n$. Also the superscript " $r d$ " indicates reaction depletion by surface flux. Again, the left subscript index $i$ represents the flux species $(i=a o, s$, and $h c)$, and the right subscript index $j$ represents the active surface species $(j=a o, s, h c)$.

\subsection{Chemical reaction}

For simplicity of modeling, only chemical reaction between $\mathrm{AO}$ and silicone are considered. Thus the remaining processes affecting surface coverage are: (1) chemical reactions due to collisions with the $\mathrm{AO}$ and silicone surface constituents by the opposite member's flux and (2) chemical reactions due to surface reactions between these two species at adjacent sites. The flux-on-surface reactions are treated similarly to the adsorption and depletion processes given by equations [5] and [7], respectively. Hence, the fraction of sites converted in a unit time by reactions of type (1) can be written as

$$
G_{i-j}^{r g}=1-e^{-\left(m_{i} P_{i-j}^{r g} / n\right)}
$$

The superscript "rg" indicates reactions forming glass, and the subscript indices denote the species AO or silicone, with the flux component being the left index. The type (2) chemical reactions are taken to be proportional to the product of the surface concentrations. This proposed model seems reasonable. A more general approach would be to use the product of the concentrations raised to powers that are characteristic of the reaction and consistent with the experimental behavior; the exponents of the concentrations may even be fractional. The actual reaction mechanism may be complex, involving a number of elementary reactions and reaction intermediaries. Lacking definitive values of the exponents for the overall reaction process, it is assumed that the fraction of sites undergoing surface reactions of $[\mathrm{AO}]+[\mathrm{S}] \rightarrow[\mathrm{G}]$ depends bilinearly on the surface concentrations of these two species. Hence, the surface-reaction depletion fraction, denoted by $G^{\text {rg }}$ is a nondimensional constant analogous to the rate constant:

$$
G^{r g}=k
$$

This states that a fixed fraction of the reaction species will be converted in a unit time starting from specified concentrations. Extension of the model can be made to include chemical reactions between all species types, such as AO reactions with hydrocarbons, by including the appropriate terms to account for those chemical processes.

What has been done here is to focus on only the sites occupied by reactant types, and thus the fractional gains above are relative to the sites of the respective species. To obtain the corresponding fractional specie coverage with respect to the total aggregate, the various $G$-quantities must be multiplied by their fractional representations. This is done in the next section using the appropriate weights through the $F_{i}$ and $F_{j}^{i}$.

\section{ITERATION EQUATIONS FOR SURFACE COVERAGE}

The iteration equations updating the absolute fractional coverage from the previous unit time step are now presented. This is merely a matter of scaling the relative fractions for each process, done by multiplying by the appropriate fractional weights using the $F_{i}$ and $F_{j}^{i}$.

The iteration equation for the fractional glass surface coverage, $F_{g}$, is explained for each group of terms according to process, which can be readily identified by the superscript indices. The superscripts $k$ and $k-1$ are used to denote the new and previous time levels, respectively. The remaining species equations follow similarly and no further explanation is given. Before detailing the process, it is noted that for each equation every term contains a $G$ factor except the leading term $F_{j}^{k-1} \times 1$. The occurrence of a term in an equation is balanced by the corresponding negative term in another equation. It results, as expected, that the following conservation of fractions holds between time steps: 


$$
F_{g}^{k}+F_{s}^{k}+F_{h c}^{k}+F_{a o}^{k}=F_{g}^{k-1}+F_{s}^{k-1}+F_{h c}^{k-1}+F_{a o}^{k-1}=1 .
$$

The iteration equations for the glass, silicone, hydrocarbon and atomic oxygen fractions are given by the following equations:

$$
\begin{aligned}
& F_{g}^{k}=F_{g}^{k-1}\left(1-G_{s-g}^{a}-G_{h c-g}^{a}-G_{a o-g}^{a}\right)+F_{s}^{k-1} F_{g}^{s}\left(G_{s-s}^{r d}+G_{h c-s}^{r d}+G_{a o-s}^{r d}\right) \\
& +F_{h c}^{k-1} F_{g}^{h c}\left(G_{s-h c}^{r d}+G_{h c-h c}^{r d}+G_{a o-h c}^{r d}\right)+F_{a o}^{k-1} F_{g}^{a o}\left(G_{s-a o}^{r d}+G_{h c-a o}^{r d}+G_{a o-a o}^{r d d}\right) \\
& +F_{s}^{k-1} F_{g}^{s} G_{s}^{d s}+F_{h c}^{k-1} F_{g}^{h c} G_{h c}^{d s}+F_{a o}^{k-1} F_{g}^{a o} G_{a o}^{d s}+F_{s}^{k-1} G_{a o-s}^{r g}+F_{a o}^{k-1} G_{s-a o}^{r g}+2 F_{s}^{k-1} F_{a o}^{k-1} G^{r g}, \\
& F_{s}^{k}=F_{s}^{k-1}\left(1-G_{a o-s}^{r g}-G_{h c-s}^{a}-G_{a o-s}^{a}\right)+F_{g}^{k-1} G_{s-g}^{a}+F_{h c}^{k-1} G_{s-h c}^{a}+F_{a o}^{k-1} G_{s-a o}^{a}+F_{s}^{k-1}\left(F_{s}^{s}-1\right)\left(G_{s-s}^{r d}+G_{h c-s}^{r d}+G_{a o-s}^{r d}\right) \\
& +F_{h c}^{k-1} F_{s}^{h c}\left(G_{s-h c}^{r d}+G_{h c-h c}^{r d}+G_{a o-h c}^{r d}\right)+F_{a o}^{k-1} F_{s}^{a o}\left(G_{s-a o}^{r d}+G_{h c-a o}^{r d}+G_{a o-a o}^{r d}\right) \\
& +F_{s}^{k-1}\left(F_{s}^{s}-1\right) G_{s}^{d s}+F_{h c}^{k-1} F_{s}^{h c} G_{h c}^{d s}+F_{a o}^{k-1} F_{s}^{a o} G_{a o}^{d s}-F_{s}^{k-1} F_{a o}^{k-1} G^{r g}, \\
& F_{h c}^{k}=F_{h c}^{k-1}\left(1-G_{s-h c}^{a}-G_{a o-h c}^{a}\right)+F_{g}^{k-1} G_{h c-g}^{a}+F_{s}^{k-1} G_{h c-s}^{a}+F_{a o}^{k-1} G_{h c-a o}^{a}+F_{s}^{k-1} F_{h c}^{s}\left(G_{s-s}^{r d}+G_{h c-s}^{r d}+G_{a o-s}^{r d}\right) \\
& +F_{h c}^{k-1}\left(F_{h c}^{h c}-1\right)\left(G_{s-h c}^{r d}+G_{h c-h c}^{r d}+G_{a o-h c}^{r d}\right)+F_{a o}^{k-1} F_{h c}^{a o}\left(G_{s-a o}^{r d}+G_{h c-a o}^{r d d}+G_{a o-a o}^{r d d}\right) \\
& +F_{s}^{k-1} F_{h c}^{s} G_{s}^{d s}+F_{h c}^{k-1}\left(F_{h c}^{h c}-1\right) G_{h c}^{d s}+F_{a o}^{k-1} F_{h c}^{a o} G_{a o}^{d s}, \\
& F_{a o}^{k}=F_{a o}^{k-1}\left(1-G_{s-a o}^{r g}-G_{s-a o}^{a}-G_{h c-a o}^{a}\right)+F_{g}^{k-1} G_{a o-g}^{a}+F_{s}^{k-1} G_{a o-s}^{a}+F_{h c}^{k-1} G_{a o-h c}^{a}+F_{s}^{k-1} F_{a o}^{s}\left(G_{s-s}^{r d}+G_{h c-s}^{r d}+G_{a o-s}^{r d}\right) \\
& +F_{h c}^{k-1} F_{a o}^{h c}\left(G_{s-h c}^{r d}+G_{h c-h c}^{r d}+G_{a o-h c}^{r d}\right)+F_{a o}^{k-1}\left(F_{a o}^{a o}-1\right)\left(G_{s-a o}^{r d}+G_{h c-a o}^{r d d}+G_{a o-a o}^{r d}\right) \\
& +F_{s}^{k-1} F_{a o}^{s} G_{s}^{d s}+F_{h c}^{k-1} F_{a o}^{h c} G_{h c}^{d s}+F_{a o}^{k-1}\left(F_{a o}^{a o}-1\right) G_{a o}^{d s}-F_{s}^{k-1} F_{a o}^{k-1} G^{r g} .
\end{aligned}
$$

In discussing the terms in equation [11], the terms are examined from left to right. The first terms involve the adsorption of silicone, hydrocarbon and $\mathrm{AO}$ onto glass. The increment to absolute fraction of glass by adsorption is negative due to loss of glass fraction. The increment equals the product resulting from multiplying the sum of the three $G_{i-g}^{a}$ terms by the absolute fractional area of glass, $F^{k-1}{ }_{g}$ existing at the previous time step. The second category comprises three groups of three terms. These consist of the reaction depletion caused by atomic and molecular flux dislodging species from their sites. Since it is assumed that silica sites are not removable, the terms are positive here due to this process. The first set of three depletion terms is the set due to silicone being removed by silicone, hydrocarbon, and AO flux, respectively. The absolute fraction of glass surface gained is dependent proportionally on the absolute fraction of silicone, $F^{k-1}{ }_{s}$, times the relative fraction of this silicone fraction that covers glass, $F_{g}^{s}$. Thus, the product of these factors, multiplied by the sum of the three depletion terms in the silicone group, gives the increment due to silicone depletion. The two remaining depletion groups involve the hydrocarbon and AO site fractions, and they follow similarly by using the appropriate multiplicative fractions. The third group consists of three terms due to desorption. The first of these involves desorption of silicone. The absolute fractional gain 
in glass area due to silicone desorption is equal to the triple product of the absolute fraction of silicone, $F^{k-1}$, times the relative fraction of silicone that covers glass, $F_{g}^{s}$, times the fraction of silicone desorbed in the time period, $G^{d s}$. The two desorption terms relating to hydrocarbon and $\mathrm{AO}$ follow similarly. The fourth set of terms represent the creation of glass area by silicone and $\mathrm{AO}$ flux and are positive terms. These involve the $G^{r g}{ }_{a o-s}$ and $G^{r g}{ }_{s-a o}$ relative fractions, which must be multiplied, respectively, by $F_{s-1}^{k-1}$ and $F^{k-1}{ }_{a o}$ representing the absolute fractions for the surfaces struck by the opposite type flux. The last group consists of the singleton term due to surface reaction of silicone and AO. It is equal to the product of the

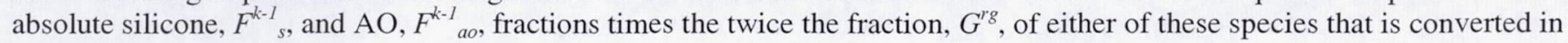
a time step.

\section{ITERATION EQUATIONS FOR SUB-LEVEL COVERAGE}

The iteration equations (with $\mathrm{k}$ denoting the time-step level) for the glass, silicone, hydrocarbon and atomic oxygen absolute fractions, $f_{j}^{i}$, are given by the following equations:

\subsection{Glass on glass}

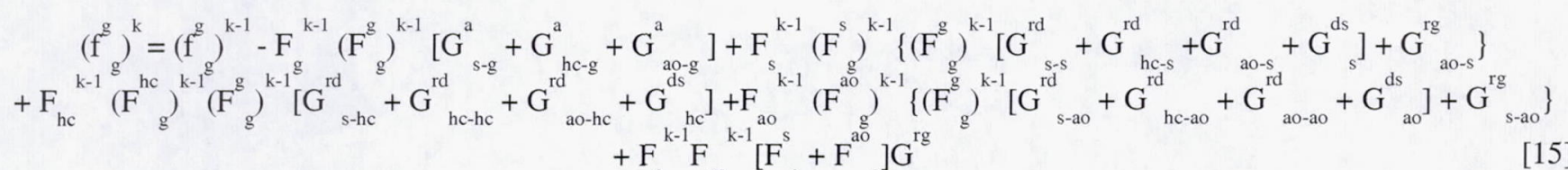

\subsection{Glass on silicone}

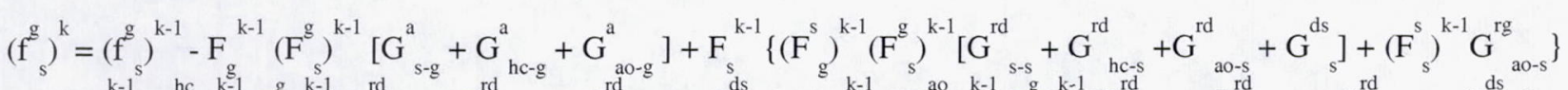

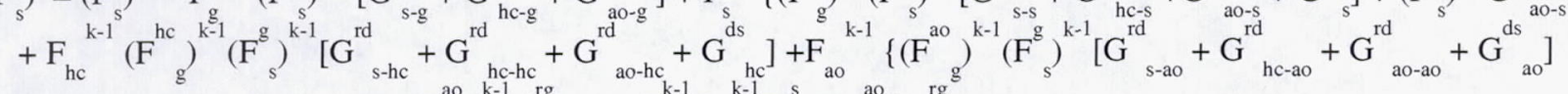

$$
\begin{aligned}
& \left.+\left(F_{s}^{a o}\right)^{k-1} G_{s-a 0}^{r g}\right\}+F_{s}^{k-1} F_{\text {ao }}^{k-1}\left[F_{s}^{s}+F_{s}^{\text {ao }}{ }_{s} G^{r g}\right.
\end{aligned}
$$

\subsection{Glass on hydrocarbon}

$$
\begin{aligned}
& \left(f_{h c}^{g}\right)^{k}=\left(f_{h c}^{g}\right)^{k-1}-F_{g}^{k-1}\left(F_{h c}^{g}\right)^{k-1}\left[G_{s-g}^{a}+G_{h c-g}^{a}+G_{a 0-g}^{a}\right]+F_{s}^{k-1}\left\{\left(F_{g}^{s}\right)^{k-1}\left(F_{h c}^{g}\right)^{k-1}\left[G_{s-s}^{\text {rd }}+G_{k-1}^{\text {rd }}{ }_{h c-s}+G_{\text {ao-s }}^{\text {rd }}+G^{d s}\right]+\left(F_{h c}^{s}\right)^{k-1} G^{r g}{ }_{a 0-s}\right\}
\end{aligned}
$$

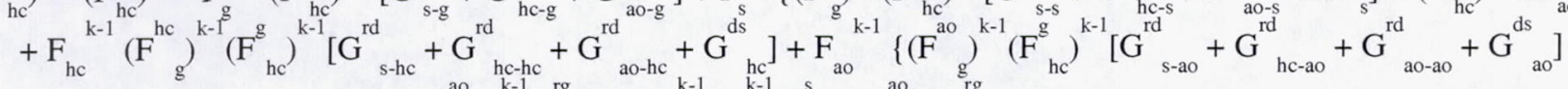

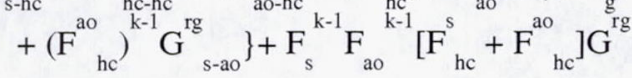

\subsection{Glass on $\mathrm{AO}$}

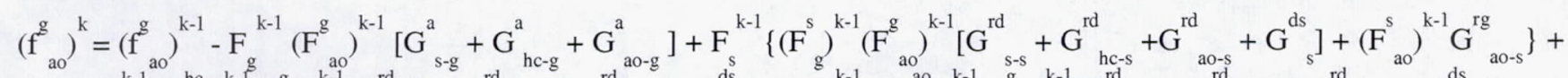

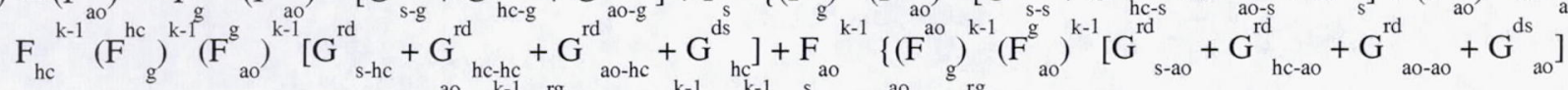

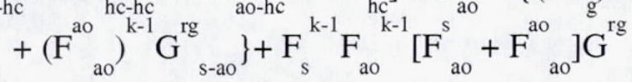

\subsection{Silicone on glass}

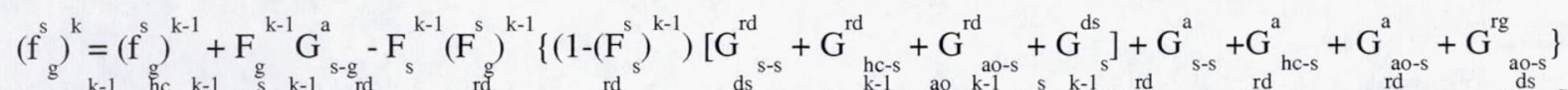

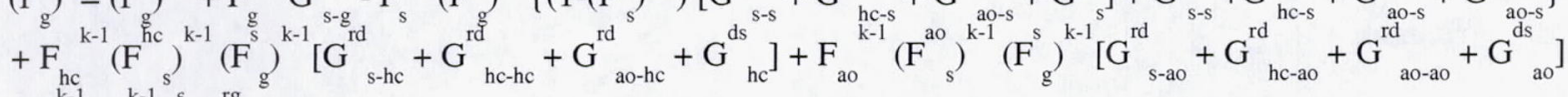

$$
\begin{aligned}
& -F_{s}^{k-1} F_{a o}^{k-1} F_{g}^{s} G^{r g}
\end{aligned}
$$


6.6 Silicone on silicone

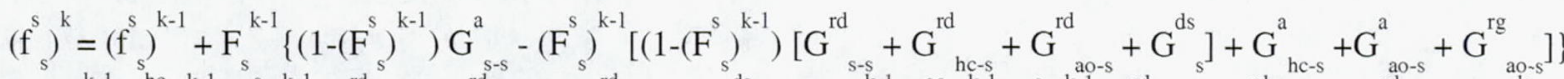

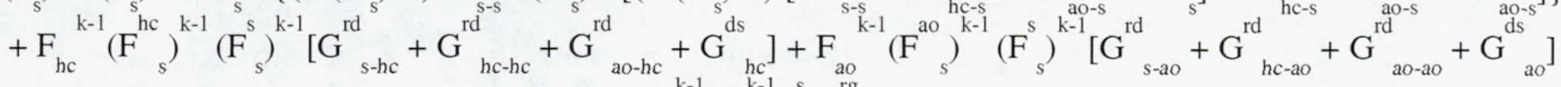

$$
\begin{aligned}
& -\mathrm{F}_{\mathrm{s}}{ }^{\mathrm{k}-1} \mathrm{~F}_{\mathrm{ao}}^{\mathrm{k}-1} \mathrm{~F}_{\mathrm{s}}^{\mathrm{s}} \mathrm{G}^{\mathrm{rg}}
\end{aligned}
$$

\subsection{Silicone on hydrocarbon}

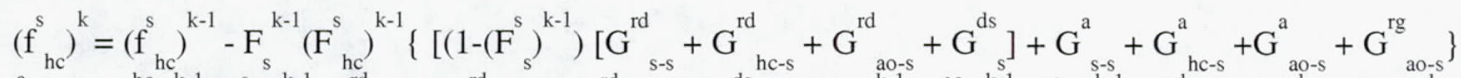

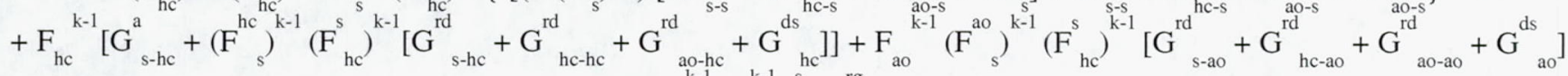

$$
\begin{aligned}
& -\mathrm{F}_{\mathrm{s}}^{\mathrm{k}-1} \mathrm{~F}_{\text {ao }}^{\mathrm{k}-1} \mathrm{~F}_{\mathrm{hc}}^{\mathrm{s}} \mathrm{G}^{\mathrm{rg}}
\end{aligned}
$$

6.8 Silicone on AO

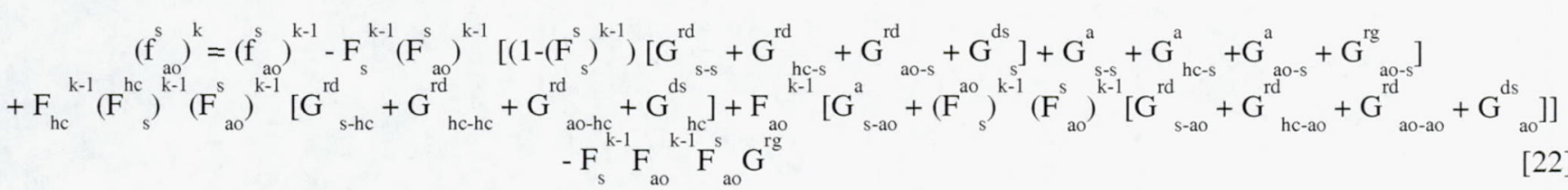

6.9 Hydrocarbon on glass

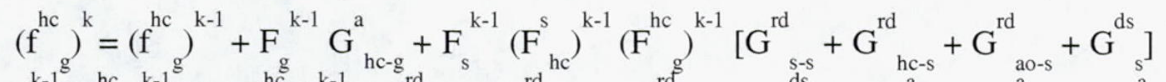

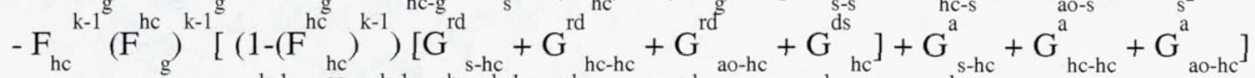

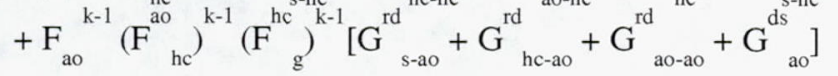

6.10 Hydrocarbon on silicone

$$
\begin{aligned}
& \left(f^{\text {he }}\right)^{k}=\left(f^{\text {he }}\right)^{k-1}+F_{s}^{k-1}\left[G_{\text {hes }}^{a}+\left(F_{h c}^{s}\right)^{k-1}\left(F_{s}^{\text {he }}\right)^{k-1}\left[G_{s-s}^{\text {rd }}+G^{\text {rd }}{ }_{\text {hc-s }}+G^{\text {rd }}{ }_{\text {ao-s }}+G^{\text {ds }}{ }_{s}\right]\right]
\end{aligned}
$$

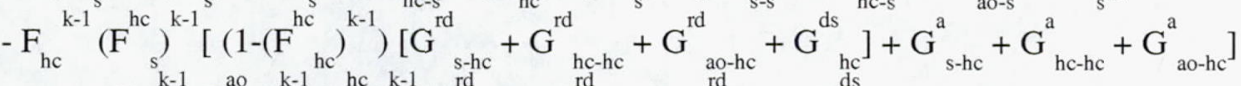

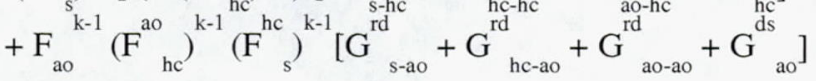

\subsection{Hydrocarbon on hydrocarbon}

$$
\begin{aligned}
& \left(f_{h c}^{h c}\right)^{k}=\left(f_{h c}^{h c}\right)^{k-1}+F_{s}^{k-1}\left(F_{h c}^{s}\right)^{k-1}\left(F_{h c}^{h c}\right)^{k-1}\left[G^{\text {rd }}{ }_{s-s}+G^{\text {rd }}{ }_{h c-s}+G^{\text {rd }}{ }_{\text {ao-s }}+G^{d s}{ }_{s}\right] \\
& +F_{h c}^{k-1}\left\{\left(1-\left(F_{h c}^{h c}\right)^{k-1}\right) G_{h c-h c}^{a}-\left(F_{h c}^{h c}\right)^{k-1}\left[\left(1-\left(F_{h c}^{h c}\right)^{k-1}\right)\left[G_{\text {s-hc }}^{\text {rd }}+G_{h c-h c}^{\text {rd }}+G^{\text {rd }}{ }_{\text {ao-hc }}+G^{d s}{ }_{h c}\right]\right.\right.
\end{aligned}
$$

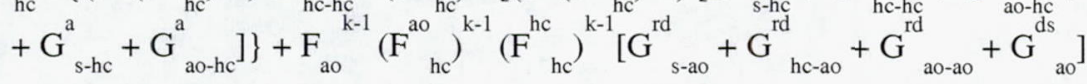

\subsection{Hydrocarbon on AO}

$$
\begin{aligned}
& \left(f^{\text {he }}{ }^{k}=\left(f^{\text {hc }}{ }^{k-1}+F_{s}^{k-1}\left(F^{s}\right)^{k-1}\left(F^{\text {hc }}\right)^{k-1}\left[G^{\text {rd }}+G^{\text {rd }}{ }_{\text {hes }}+G^{\text {rd }}{ }^{\text {ace }}+G^{\text {ds }}\right]\right.\right.
\end{aligned}
$$

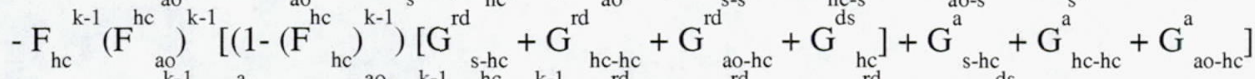

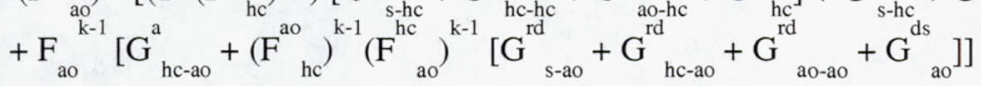




\subsection{AO on glass}

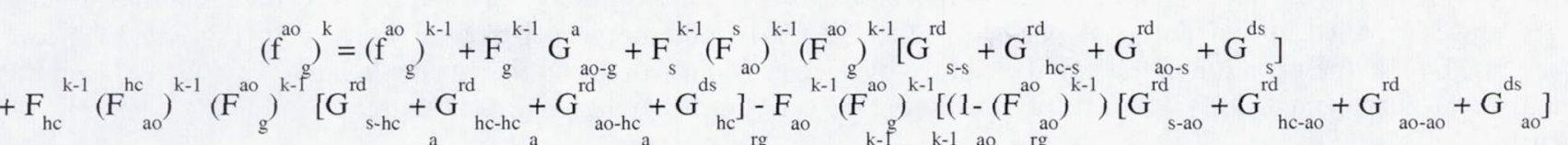

$$
\begin{aligned}
& +\mathrm{G}_{\text {s-hc }}^{\mathrm{a}}+\mathrm{G}_{\text {hc-ao }}^{\mathrm{a}}+\mathrm{G}_{\text {ao-ao }}^{\mathrm{a}}+\mathrm{G}_{\mathrm{s}-\mathrm{ao}}^{\mathrm{rg}}{ }^{\mathrm{ao}}-\mathrm{F}_{\mathrm{s}}^{\mathrm{k}-\mathrm{f}} \mathrm{F}_{\text {ao }}^{\mathrm{k}-1} \mathrm{~F}_{\mathrm{g}}^{\mathrm{ao}} \mathrm{G}^{\mathrm{rg}}
\end{aligned}
$$

\subsection{AO on silicone}

$$
\begin{aligned}
& \left(f_{s}^{\text {ao }}\right)^{k}=\left(f_{s}^{\text {ao }}\right)^{k-1}+F_{s}^{k-1}\left[G_{\text {ao-s }}^{\text {a }}+\left(F_{\text {ao }}^{s}\right)^{k-1}\left(F_{s}^{\text {ao }}\right)^{k-1}\left[G_{s-s}^{\text {rd }}+G_{\text {hc-s }}^{\text {rd }}+G_{\text {ao-s }}^{\text {rd }}+G_{s}^{\text {ds }}\right]\right.
\end{aligned}
$$

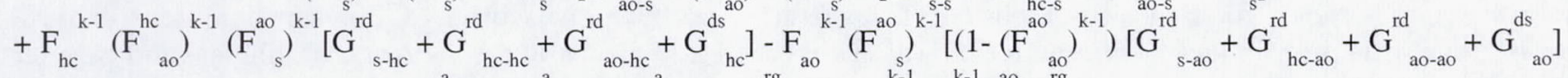

$$
\begin{aligned}
& \left.+\mathrm{G}_{\text {s-ao }}^{\mathrm{a}}+\mathrm{G}_{\text {hc-ao }}^{\mathrm{a}}+\mathrm{G}_{\text {ao-ao }}^{\mathrm{a}}+\mathrm{G}_{\text {s-ao }}^{\mathrm{rg}}\right]-\mathrm{F}_{\mathrm{s}}^{\mathrm{k}-1} \mathrm{~F}_{\text {ao }}^{\mathrm{k}-1} \mathrm{~F}_{\mathrm{s}}^{\mathrm{ao}} \mathrm{G}^{\mathrm{rg}}
\end{aligned}
$$

6.15 AO on Hydrocarbon

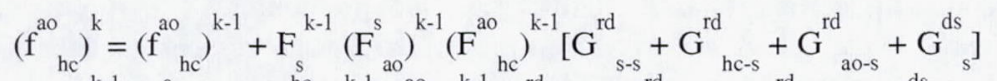

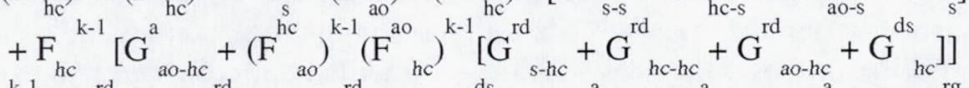

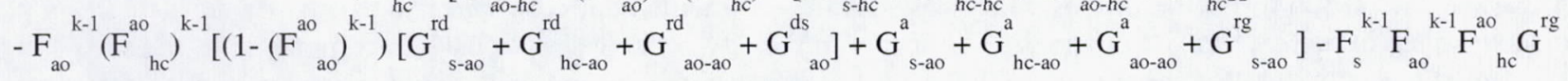

\subsection{6 $\mathrm{AO}$ on $\mathrm{AO}$}

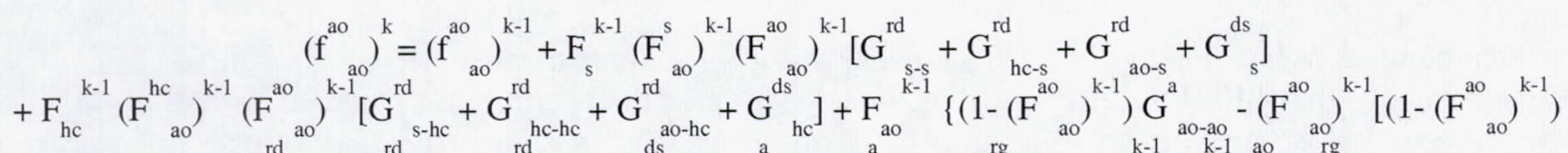

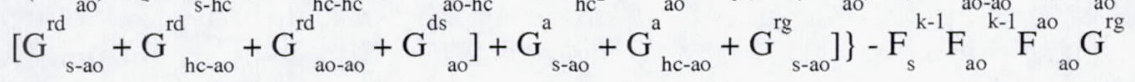

These updated absolute fractions, $f_{j}^{i}$, are used in computing new relative fractions, $F_{j}^{i}$, by using Equation 3 .

\section{RESULTS OF MODELING}

For the purpose of examining the essential features of film growth, a prototype code was constructed using a popular spreadsheet computer program to simulate the processes of adsorption, desorption, depletion, and chemical reaction. Using this code, $\mathrm{SiO}_{x}$ growth can be modeled based on the relative fluxes of $\mathrm{AO}$, hydrocarbon, and silicone. By knowing the amount of contaminants trapped during the film growth, the resulting optical transmittance of the contaminated film can be modeled based on assumed values of absorption coefficients. The preliminary results reported here are based on simple models that are hoped to be useful in explaining results from in-space and ground-based contamination. A brief review of some recent findings concerning both in-space and ground-based contamination is presented before discussing results from this present study.

In-space contamination results ${ }^{5}$ indicate that the flux of volatile components of materials such as silicones and hydrocarbons decreases over time from the early days of a mission. From studying the variation of thickness among the different layers in films deposited on spacecraft material exposed to both contaminant and $\mathrm{AO}$ flux, it was concluded that variations in the rate of oxidation occurred. The thickness of the layers decreased from the inner layers to the outer layers of the films. Variations in the degree of oxidation were also determined by examining surfaces exposed to different rates of contaminant flux. The oxidation was determined to vary with respect to the rate of silicone and hydrocarbon arrival because a much thinner, more fully oxidized and more transparent contaminant layer was formed on the surfaces believed to have received lower silicone and hydrocarbon flux. Ground-based results ${ }^{5}$ support the conclusion that small fluxes of contaminants relative to a fixed AO flux produce a nearly fully oxidized film, whereas large ratios of contaminant to AO flux result in markedly reduced oxidization fractions. 
A test case is presented using the numerical code and is compared to the qualitative results observed experimentally. The specification of a decaying contaminant flux is made because, as mentioned above, the flux of volatile materials diminishes with time. An AO flux composed of periodic spikes of large amplitude superimposed on a relatively low base level is prescribed. First, the variation in fractional coverage by species is discussed, and then a simple model of optical transmittance degradation is presented along with a result illustrating the effect of the AO-to-silicone flux ratio.

The distributions of the silicone and hydrocarbon fluxes for the test are shown in figure 3 as solid curves. The AO flux, having a peak relative amplitude of one, is shown as the dotted curve. Based on these fluxes, a somewhat arbitrary or hypothetical specification of values for the various physical quantities such as sticking probability was made. The actual values used are values chosen as being representative in the sense that they are reasonable, but not necessarily and probably not correct. For example, a sticking probability equal to 0.1 may be reasonable for a single gas-solid specification but not likely the correct choice for all species, substrate temperatures, and fractional coverage. For simplicity in specification, essentially uniform probabilities were used for the various processes. In any case, the various probabilities must be selected in conjunction with the prescribed fluxes so as to limit the change in fractional surface areas during a unit time step. Although not mentioned previously, the procedure relies on the assumption that the processes can be treated independently. This will not be the case if the average site is being multiply struck in a single time-step increment. This restriction is easily met by using relatively small $m$-to- $n$ ratios. For such a set of hypothetical probabilities, figure 4 shows the variation in surface coverage by species corresponding to the relative fluxes presented previously. The top curve represents the fractional coverage of glass starting from a clean silica surface. From this figure, it can be seen that the glass fractional area initially declines quickly as the other species populate through adsorption. The hydrocarbon and silicone fluxes increase steadily at first, peak and then begin to decline in coverage. This decline in contaminant coverage is balanced by an increase in glass coverage and a slight increase in AO coverage. The jumps in the AO coverage, obviously corresponding to the spikes in the AO flux, are balanced by almost equally sharp falls in the hydrocarbon coverage and silicone coverage, with slight drops in the glass coverage. Variation of parameters about values used in this example produce reasonable changes in the fractional coverage by species.

One important component contributing to the fractional glass coverage is that which accrues due to the chemical reaction processes associated with the final three terms of Equation [11]. These three terms are the source for film growth. The first two terms represent the fractional increase in glass by chemical reactions resulting from flux of type $i$ on surface type $j$, where the species type is either silicone or AO. The third of these three terms represents the fractional increase in glass due to chemical reactions occurring at adjacent silicone and AO surface sites. A simple modeling of the contaminant film would be to use a linear combination of the three terms while accounting for trapping of the contaminants. To simplify the discussion, the choice of using only the last term of the three is made here and, furthermore, the assumption is made that trapping of contaminants is proportional to this term. Using the fractional coverage provided in the code, the contaminant thickness can be integrated numerically. The relative transmittance is calculated based on the Beer-Lambert law, which is given, assuming the absorbing material is distributed uniformly throughout the film, by

$$
\tau=e^{-\alpha L}
$$

where $\alpha$ is the optical absorption coefficient and $L$ is the integrated film thickness, which generally increases nonlinearly with time. The time variation of relative transmittance of a contaminant film produced using the fluxes as prescribed above is presented in figure 5 as the solid curve. The rate of reduction in transmittance increases significantly during intervals of peak AO flux. The exponential-like decline in transmittance is due to the exponentially declining silicone flux. The variation of transmittance is also shown for the same case but where the AO flux is prescribed as being a constant, equal to the timeaveraged value of the spiked AO-flux distribution. The transmittance decline seen for the constant AO-flux case is slightly less than the spiked AO-flux case, otherwise it nearly serves as an upper envelope for the spiked AO-flux transmittance curve. The greater overall decline in transmittance for the spiked AO-flux distribution can be explained by noting that the early large spikes in AO flux provide the oxidizer needed to combine with the high initial contaminant flux. This produces a rapid decline in transmittance that is never again matched. For "real" values of the parameters, this would indicate that the most significant contaminant degradation may occur relatively early in a spacecraft mission. It must be remembered that the values used here for the probabilities of adsorption and reaction, etc., are reasonable hypothetical values and are mainly used as placeholders to check out the mechanics and the features of the code. The correct values of the process parameters must come from either experiment or thermodynamic analysis. 


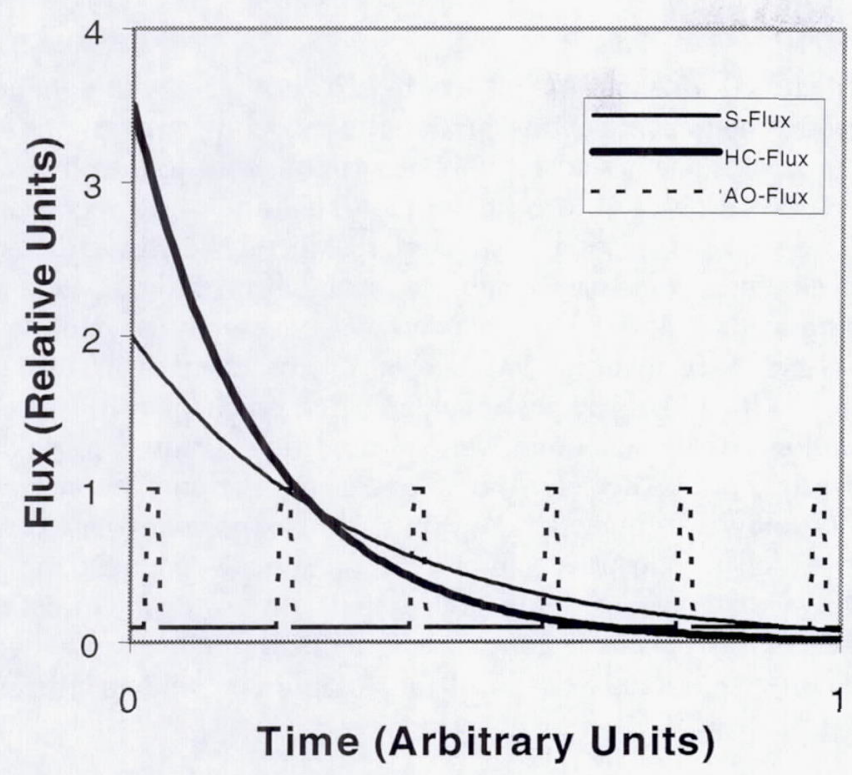

Fig. 3 - Variation of species flux with time.

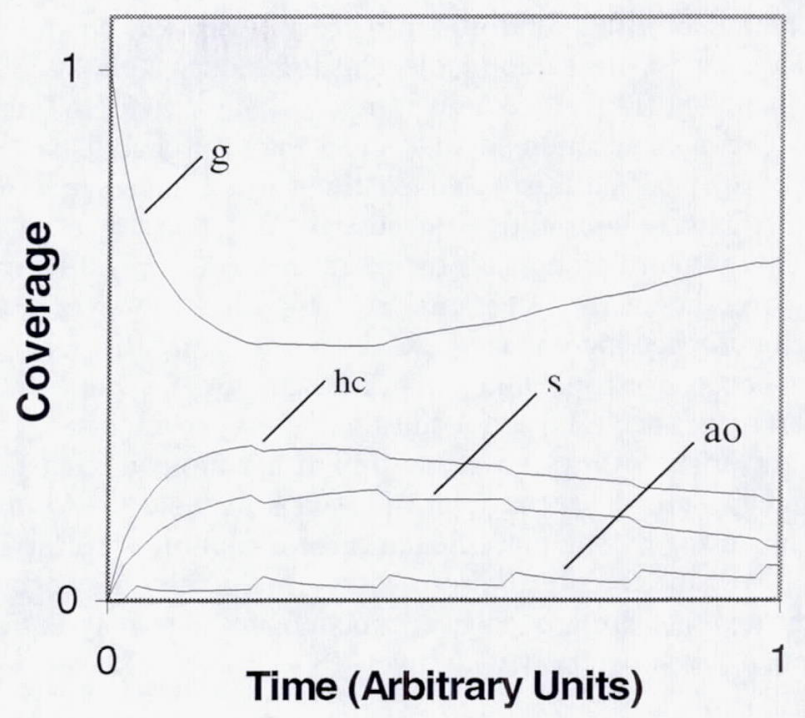

Fig. 4 - Evolution of fractional coverage for species.

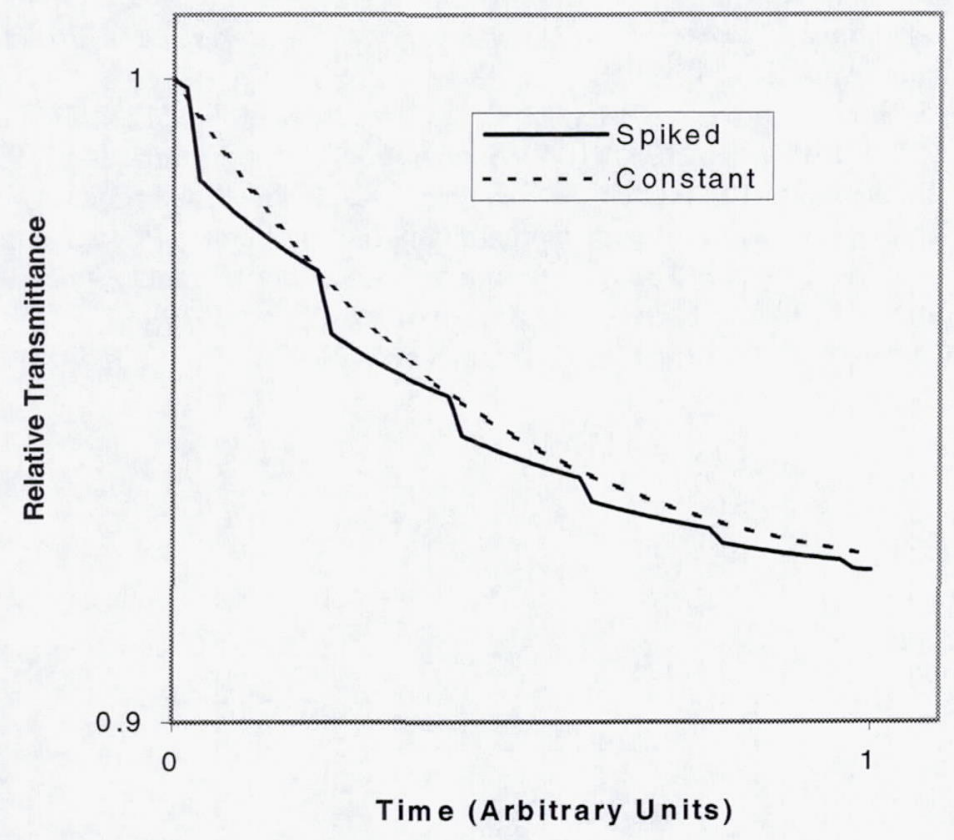

Fig. 5 - Relative optical transmittance versus time. 


\section{SUMMARY}

Contaminant films can form on spacecraft surfaces degrading system performance. AO present in LEO can react with volatile silicone and hydrocarbon molecules to produce a glassy $\mathrm{SiO}_{\mathrm{x}}$ based contaminant coating. In order to model the growth of the contaminant film, procedures were devised to model the surface processes such as adsorption, desorption, collision depletion, and chemical reaction. It was found that a probabilistic approach could be used to estimate the likelihood of a process in terms of specified fluxes and existing surface coverage. Four specie types, consisting of silica glass, silicones, hydrocarbons, and $\mathrm{AO}$, were considered. Because the different processes involved changes in surface site occupancy, it proved convenient to conceptually group sites by specie type. A detailed bookkeeping method devised to track surface coverage by specie type allowed the surface fractional coverage and sub-layer fractional coverage to be updated based on the changes produced during fluence increments. Equations were presented for updating the fractional species coverage based on changes in specie site occupation, including a full discussion for one such equation. Also, equations were presented, according to each overlaying specie type, for the first sub-layer fractional coverage by specie. Because the values of the various parameters characteristic of a given set of flux distributions are not generally known, estimated values must be used in many cases. A standard spreadsheet program proved a suitable platform from which to construct a prototype numerical code. Example results using a code that calculates the evolution of surface coverage were presented. One example illustrates for a particular case how the fractional coverage evolves given time dependent fluxes. An example for a simple modeling of transmittance indicates that the growth in a contaminant layer may come early in a spacecraft mission if the volatile contaminant fluxes diminish exponentially with time.

\section{REFERENCES}

1. B.A. Banks, J.A. Dever, L. Gebauer, and C.M. Hill: "Atomic Oxygen Interactions with FEP Teflon and Silicones on LDEF", Proceedings of the $1^{\text {st }}$ LDEF Post-Retrieval Symposium, pp. 801-816, Kissimmee, Florida, June 2-8, 1991.

2. E.R. Crutcher and K.J. Warner, "Molecular Films associated with LDEF", Proceedings of the $1^{\text {st }}$ LDEF Post-Retrieval Symposium, pp. 155-177, Kissimmee, Florida, June 2-8, 1991.

3. Y. Noter, E. Grossman, L. Genkin, Y. Haruvey, M. Murat, I. Ross, R. Vered, Y. Lifshitz, Y. Efraty, H. Granot, A. Halsbersberg, H. Lauria, and A. Stoler, "Variations in the Telemetry of an Offeq Satellite's Sun Sensors", Proceedings of the $3^{\text {rd }}$ International Space Forum on Protection of Materials and Structures from the LEO Space Environment, Toronto, Canada, April 25-26, 1996.

4. B. Blakkolb, L.E. Ryan, H.S. Bowen and T.J. Kosic: "Optical Characterization of LDEF Contaminants", Proceedings of the $2^{\text {nd }}$ LDEF Post-Retrieval Symposium, pp. 1035-1040, San Diego, California, June 1-5, 1992.

5. B.A. Banks, S. Rutledge, E. Sechkar, T. Stueber, A. Snyder, K.K. de Groh, C. Haytas, and D. Brinker: "Issues and Effects of Atomic Oxygen Interactions with Silicone Contaminant on Spacecraft in Low Earth Orbit", Proceedings of the $8^{\text {th }}$ International Symposium on Materials in a Space Environment, Arcachon, France, June 5-9, 1991

6. G. Attard and C. Barnes, Surfaces, Chapter 1, Oxford University Press, Oxford, 1998.

7. A.W. Adamson, Physical Chemistry of Surfaces, Wiley Interscience, New York, 1990. 


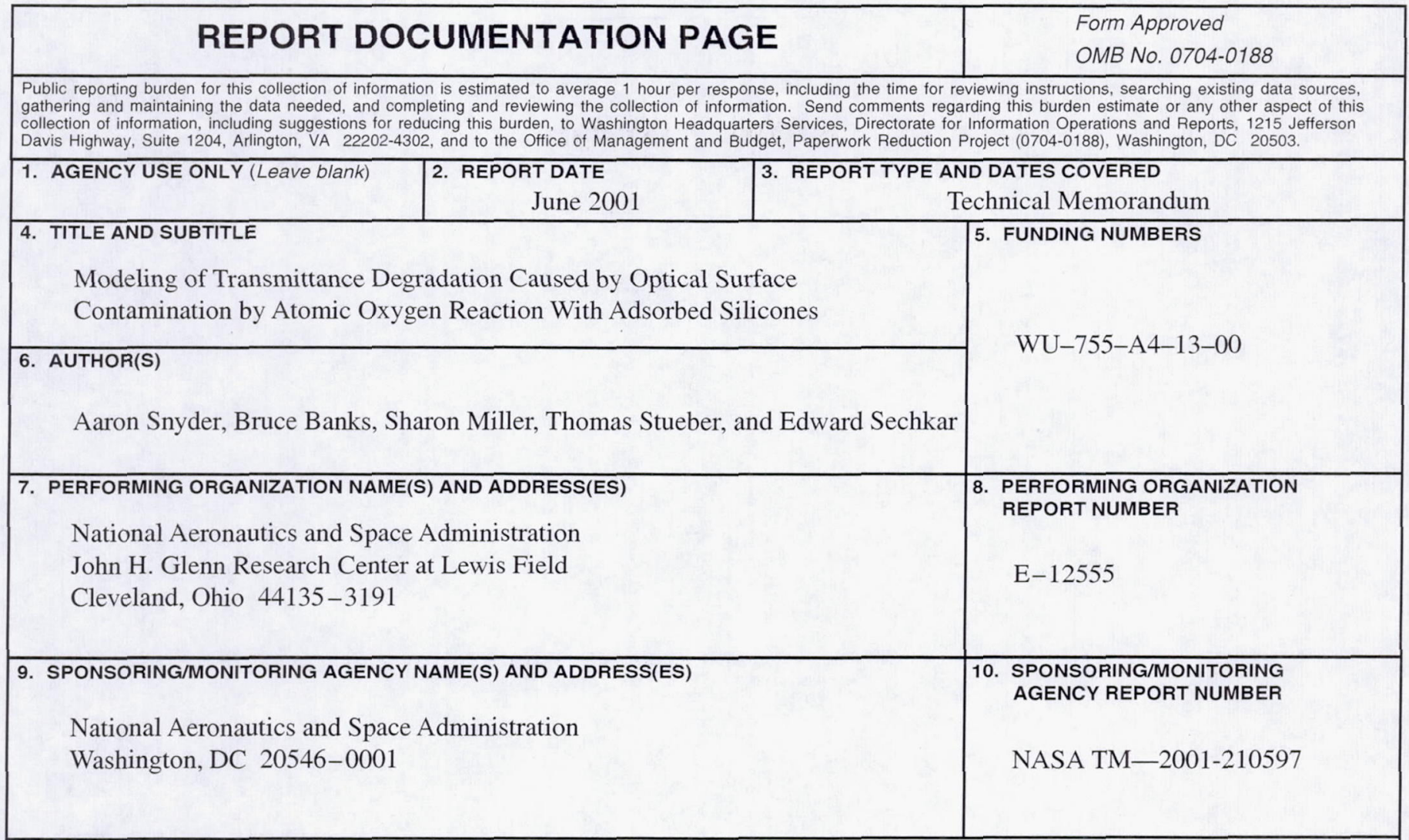

\section{SUPPLEMENTARY NOTES}

Prepared for the 45th Annual Meeting, the International Symposium on Optical Science and Technology sponsored by the International Society for Optical Engineering, San Diego, California, July 30-August 4, 2000. Aaron Snyder,

Bruce Banks, and Sharon Miller, NASA Glenn Research Center; Thomas Stueber and Edward Sechkar, Dynacs Engineering Company, Inc., 2001 Aerospace Parkway, Brook Park, Ohio 44142. Responsible person, Aaron Snyder, organization code 5480, 216-433-5918.

12a. DISTRIBUTION/AVAILABILITY STATEMENT 12b. DISTRIBUTION CODE

Unclassified - Unlimited

Subject Category: 18

Distribution: Nonstandard

Available electronically at http://gltrs.grc.nasa.gov/GLTRS

This publication is available from the NASA Center for AeroSpace Information, 301-621-0390.

13. ABSTRACT (Maximum 200 words)

A numerical procedure is presented to calculate transmittance degradation caused by contaminant films on spacecraft surfaces produced through the interaction of orbital atomic oxygen $(\mathrm{AO})$ with volatile silicones and hydrocarbons from spacecraft components. In the model, contaminant accretion is dependent on the adsorption of species, depletion reactions due to gas-surface collisions, desorption, and surface reactions between $\mathrm{AO}$ and silicone producing $\mathrm{SiO}_{\mathrm{x}}$ (where $\mathrm{x}$ is near 2). A detailed description of the procedure used to calculate the constituents of the contaminant layer is presented, including the equations that govern the evolution of fractional coverage by specie type. As an illustrative example of film growth, calculation results using a prototype code that calculates the evolution of surface coverage by specie type is presented and discussed. An example of the transmittance degradation caused by surface interaction of AO with deposited contaminant is presented for the case of exponentially decaying contaminant flux. These examples are performed using hypothetical values for the process parameters.

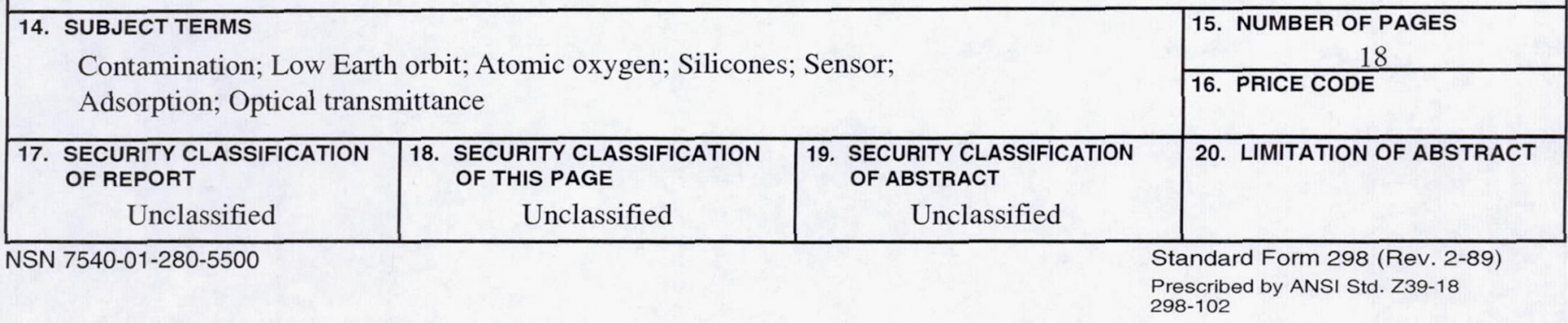

